\section{Mapping local electric fields in proteins at biomimetic interfaces $\dagger$}

\author{
Gal Schkolnik, ${ }^{a}$ Tillmann Utesch, ${ }^{a}$ Johannes Salewski, ${ }^{a}$ Katalin Tenger, ${ }^{b}$ Diego Millo, ${ }^{a}$ \\ Anja Kranich, ${ }^{a}$ Ingo Zebger, ${ }^{a}$ Claudia Schulz, ${ }^{a}$ László Zimányi, ${ }^{b}$ Gábor Rákhely, ${ }^{c}$ \\ Maria Andrea Mroginski ${ }^{a}$ and Peter Hildebrandt ${ }^{* a}$
}

Received 30th May 2011, Accepted 2nd November 2011

DOI: $10.1039 / \mathrm{c} 1 \mathrm{cc} 13186 \mathrm{a}$

We present a novel approach for determining the strength of the electric field experienced by proteins immobilised on membrane models. It is based on the vibrational Stark effect of a nitrile label introduced at different positions on engineered proteins and monitored by surface enhanced infrared absorption spectroscopy.

Most biochemical and biophysical processes of proteins take place at and in membranes and thus under the influence of electrostatic fields. Particularly strong local electric fields of the order of $10^{9} \mathrm{~V} \mathrm{~m}^{-1}$ prevail at the membrane/solution interface and in the boundary region between the hydrophobic core and the polar or charged headgroups of the membrane. ${ }^{1}$ Such high external electric fields may modulate the structure of integral membrane and membrane-attached proteins as well as the conformational and reaction dynamics of proteins, such as in enzymatic processes, ion transport, and electron transfer. ${ }^{1-7}$ Despite a large body of experimental data, no concept has yet been established to describe the electric-field dependence of protein functions on a molecular level. As an essential part of such a concept, the local electric field strengths at the protein/ membrane interface need to be quantified, which requires novel methodological strategies.

Electric field strengths may be determined on the basis of the vibrational Stark effect (VSE), which refers to the electric field induced frequency shift $\Delta \nu$ of a vibrational mode according to

$$
h c \Delta \nu=-\Delta \vec{\mu} \vec{F}
$$

where $\Delta \vec{\mu}$ is the change in the dipole moment between the vibrational ground and excited states, $\vec{F}$ is the electric field strength, and $h$ and $c$ denote the Planck constant and the speed of light, respectively. A particularly sensitive VSE reporter group is the nitrile function. In their pioneering work, Boxer and co-workers ${ }^{8-10}$ have introduced a nitrile label at different

${ }^{a}$ Technische Universität Berlin, Institut für Chemie, Sekr. PC14, Straße des 17. Juni 135, D-10623 Berlin, Germany.

E-mail: hildebrandt@chem.tu-berlin.de; Fax: +4930 31421122; Tel: + 493031421419

${ }^{b}$ Institute of Biophysics, Biological Research Centre, H-6726 Szeged Temesvári krt. 62., Hungary

${ }^{c}$ Department of Biotechnology, University of Szeged, Közép fasor 52, H-6726 Szeged, Hungary

$\dagger$ Electronic supplementary information (ESI) available: Description of experimental procedures and the MD simulation as well as further spectroscopic data on MBN. See DOI: 10.1039/c1cc13186a sites on the protein surface and at the active site of the enzyme human aldose reductase, and probed the respective $\mathrm{C} \equiv \mathrm{N}$ stretching modes of the proteins in solution. In the present work, we have, for the first time, extended this strategy to proteins immobilised on simple membrane models monitoring the $\mathrm{C} \equiv \mathrm{N}$ stretchings using surface enhanced infrared absorption (SEIRA) spectroscopy.

We have chosen the heme protein cytochrome c (Cyt-c) as a convenient test protein that can be readily electrostatically bound to $\mathrm{Au}$ electrodes coated with self-assembled monolayers (SAMs) of carboxyl-terminated alkylthiols. Such devices may be considered as a simple model for biological membranes, specifically with respect to the electrostatics at the SAM/ solution (membrane/solution) interface. ${ }^{7}$ Furthermore, the structure and reaction dynamics of Cyt-c have been shown to depend sensitively on the interfacial electric field upon binding to SAM-coated electrodes and liposomes. It has been proposed that electric field variations at the mitochondrial membrane may contribute to the switching of the protein function from electron transport in the respiratory chain to lipid peroxidation as one of the first steps in apoptosis. ${ }^{7}$ Thus, this work constitutes the starting point for a comprehensive analysis of the electric field control of Cyt-c's function.

4-Mercaptobenzonitrile (MBN) has been used as a nitrile label, covalently attached to a Cys side chain of site-directed engineered horse heart Cyt-c variants. In this work, we have introduced a Cys at two different positions, one in the vicinity of the redox centre (Lys8) and one at a relatively remote position (Lys39). The two variants, K8C and K39C, were then treated with MBN such that the aromatic nitrile was selectively bound to the thiol function of the cysteine (see ESI $\dagger$ for experimental details).

IR spectra of the labelled Cyt-c variants in solution display weak but clearly identifiable peaks in an optical window between 2200 and $2300 \mathrm{~cm}^{-1}$ that is free of any interference by IR bands of the protein, but which includes a strong background absorption (Fig. S1, ESI $\dagger$ ). To determine the frequencies of the nitrile stretching modes with higher precision, we have thus used the second derivatives of the spectra in which the minima correspond to the peak maxima of the IR absorption bands (Fig. 1 and 2; for details of the spectra analysis, see $\mathrm{ESI} \dagger$ ). For the labelled K39C variant in solution (Fig. 1, top), the nitrile stretching mode is observed at $2235.1 \mathrm{~cm}^{-1}$. 


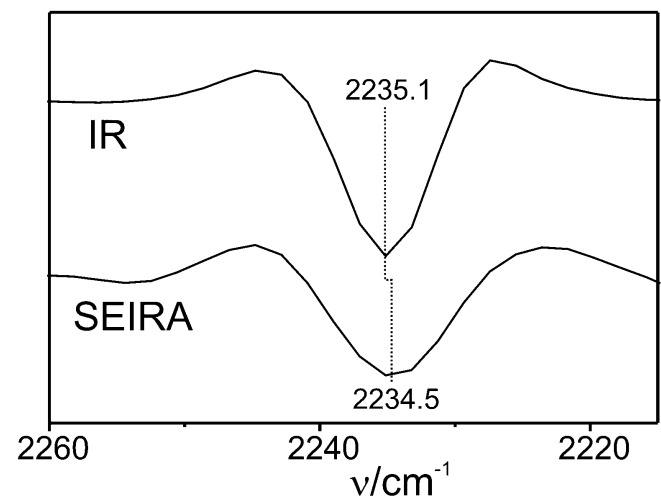

Fig. 1 Second derivatives of the IR (top) and SEIRA (bottom, measured at open circuit) spectra of the MBN-labelled K39C variant of Cyt-c. The root-mean standard deviation was \pm 0.15 (IR) and $\pm 0.4 \mathrm{~cm}^{-1}$ (SEIRA).

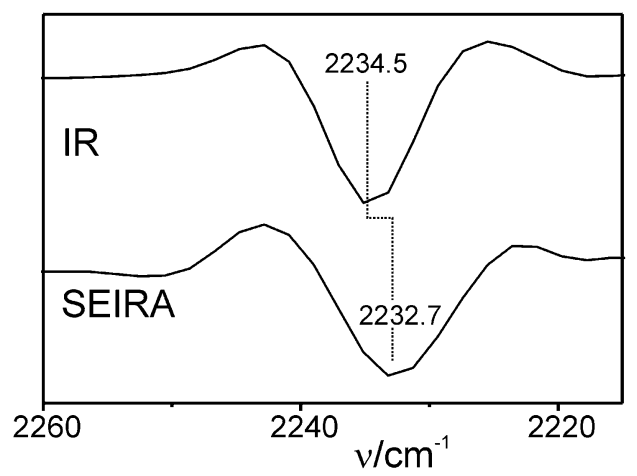

Fig. 2 Second derivatives of the IR (top) and SEIRA (bottom; measured at open circuit) spectra of the MBN-labelled K8C variant of Cyt-c. The root-mean standard deviation was \pm 0.15 (IR) and $\pm 0.9 \mathrm{~cm}^{-1}$ (SEIRA).

This frequency, which is distinctly higher than that of solid MBN $\left(2225.7 \mathrm{~cm}^{-1}\right)$ or MBN dissolved in DMSO $\left(2228.3 \mathrm{~cm}^{-1}\right.$, Fig. S2, ESI $\dagger$ ), is indicative of a hydrogen bonded nitrile group. ${ }^{11,12}$ This observation is in line with the results of molecular dynamics (MD) simulations which predict that the nitrile group is solvent-exposed and, therefore, involved in hydrogen bonding interactions with water molecules (Fig. 3). For the labelled $\mathrm{K} 8 \mathrm{C}$ variant in solution (Fig. 2, top), the $\mathrm{C} \equiv \mathrm{N}$ stretching mode is found at $2234.5 \mathrm{~cm}^{-1}$, in the same region as that measured for the $\mathrm{K} 39 \mathrm{C}$ protein variant, consistent with a solvent-exposed nitrile group. Also in this case, MD simulations predict a solution exposed nitrile group.

In addition to hydrogen bonding interactions, the stretching mode of a nitrile group attached to a protein is affected by the electrostatic field caused by charged and polar residues in its immediate environment. Since in both protein variants the nitrile label is solvent exposed and thus subject to similar hydrogen bonding interactions, the frequency difference $\Delta \nu_{\mathrm{K} 39 \mathrm{C}-\mathrm{K} 8 \mathrm{C}}$ of the respective bands at $2235.1 \mathrm{~cm}^{-1}$ (K39C) and $2234.5 \mathrm{~cm}^{-1}$ (K8C) is likely to arise mainly from the difference in the component of the protein's intrinsic electric field $\Delta F_{\mathrm{K} 39 \mathrm{C}-\mathrm{K} 8 \mathrm{C}}$ along the $\mathrm{C} \equiv \mathrm{N}$ bond, corresponding to the direction of $\Delta \vec{\mu} .^{11}$ Thus, one obtains

$$
\Delta \nu_{\mathrm{K} 39 \mathrm{C}-\mathrm{K} 8 \mathrm{C}}=\nu_{\mathrm{K} 39 \mathrm{C}}-\nu_{\mathrm{K} 8 \mathrm{C}}=-\Delta \mu_{\mathrm{STR}} \Delta F_{\mathrm{K} 39 \mathrm{C}-\mathrm{K} 8 \mathrm{C}}
$$

where $\Delta \mu_{\mathrm{STR}}$ is the Stark tuning rate. The value of $\Delta \mu_{\mathrm{STR}}$ for the $\mathrm{C} \equiv \mathrm{N}$ stretching of MBN has been previously determined
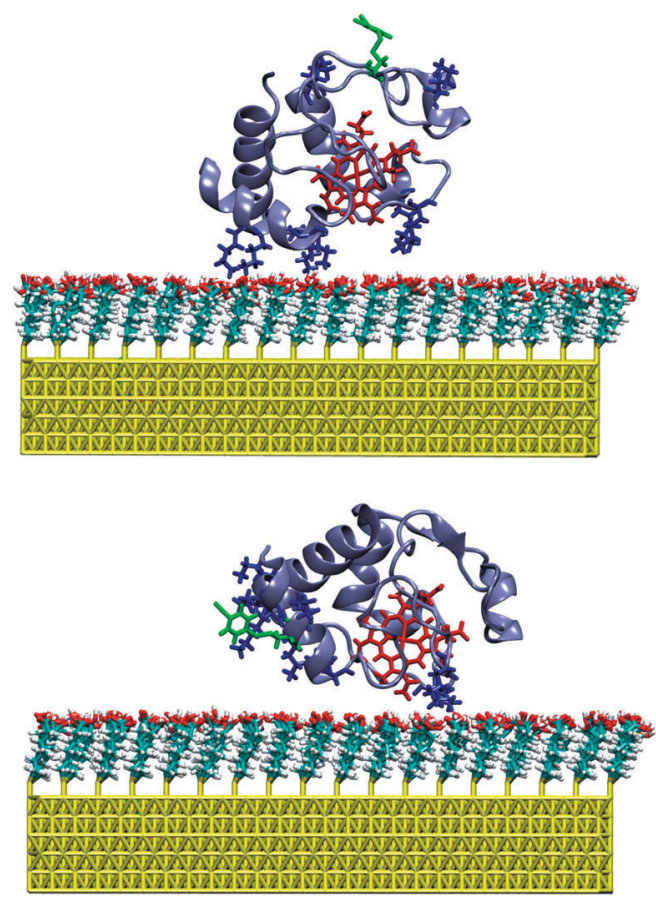

Fig. 3 MD snapshot of the labelled K39C (top) and K8C (bottom) variants of Cyt-c bound to the SAM-coated Au surface. The MBN group and the cationic lysine residues are indicated in green and blue, respectively.

to be $\Delta \mu=-7.4 \times 10^{-7} \mathrm{~cm}^{-1} / \mathrm{V} \mathrm{cm}{ }^{-1} .9$ According to eqn (2), one obtains $\Delta F_{\mathrm{K} 39 \mathrm{C}-\mathrm{K} 8 \mathrm{C}}=8.1 \times 10^{7} \mathrm{~V} \mathrm{~m}^{-1}$. This difference in the local electric field may be mainly related to the larger number of positively charged lysine residues in the vicinity of position 8 .

In the next step, the labelled proteins were immobilized on an Au film functionalised with a SAM of 6-mercaptohexanoic acid. The Au film, deposited on a silicon prism, serves as a working electrode as well as for enhancing the IR signal in an attenuated total reflection (ATR) SEIRA setup ${ }^{13}$ (see ESI $\dagger$ for further details). The negatively charged SAM allows for the electrostatic immobilization of Cyt-c via its positively charged lysine-rich binding domain surrounding the exposed heme crevice. ${ }^{7,13,14}$ Under these immobilization conditions the native protein structure and function are preserved as demonstrated by cyclic voltammetry and SEIRA spectroscopy ${ }^{13}$ (ESI $\dagger$, Fig. S3). In the SEIRA spectrum of the labelled K39C variant, measured at open circuit, the $\mathrm{C} \equiv \mathrm{N}$ stretching mode is observed at $2234.5 \mathrm{~cm}^{-1}$, i.e. at a frequency slightly lower than for the protein in solution (Fig. 1), whereas the SEIRA spectrum of the labelled $\mathrm{K} 8 \mathrm{C}$ displays a band at a distinctly lower frequency, i.e. $2232.7 \mathrm{~cm}^{-1}$ (Fig. 2).

MD simulations were carried out for the labelled K39C and $\mathrm{K} 8 \mathrm{C}$ variants immobilized on a SAM-coated $\mathrm{Au}$ surface, starting with an initial configuration in which the proteins' dipole moments were oriented perpendicularly to the surface. This set-up is expected to afford the energetically most favourable orientation of the electrostatically bound Cyt-c on the SAM-coated Au electrode. ${ }^{15,16}$ The results indicate that, as for the proteins in solution, the nitrile function remains solvent exposed in both Cyt-c variants. Furthermore, the MD simulations show no indication for immobilization-induced structural 
changes of the protein in the vicinity of the label (ESI $\dagger)$. In addition, for $\mathrm{K} 8 \mathrm{C}$ the simulations rule out a direct interaction of the nitrile function with the carboxyl groups of the SAM, even though the $\mathrm{CN}$ label is located in the vicinity of the protein binding domain (Fig. 3). Thus, one may assume that the frequency changes of the nitrile stretching observed upon binding of the proteins to the SAM surface largely result from the modulation of the local electrostatic field by the potential drop across the electrode/SAM/solution interface.

Correspondingly, one may relate the difference between the IR and SEIRA frequencies $\Delta \nu$ to the external electric field $\vec{F}(x)$ at a distance $x$ from the SAM surface according to

$$
\Delta \nu=\nu_{\text {SEIRA }}-\nu_{\text {IR }}=\left|\Delta \mu_{\text {STR }}\right||\vec{F}(x)| \cos (\alpha)
$$

Here $\alpha$ is the angle between $\vec{F}(x)$ and $\Delta \vec{\mu}$, corresponding to the angle between the $\mathrm{C} \equiv \mathrm{N}$ bond and the surface normal. Thus, the frequency differences $\Delta \nu$ of $-0.6 \mathrm{~cm}^{-1}$ (K39C) and $-1.8 \mathrm{~cm}^{-1}$ (K8C) allow determining the quantity $|\vec{F}(x)|$. $\cos (\alpha)$ according to eqn (3), yielding $-8.1 \times 10^{7} \mathrm{~V} \mathrm{~m}^{-1}$ and $-2.4 \times 10^{8} \mathrm{~V} \mathrm{~m}^{-1}$ for $\mathrm{K} 39 \mathrm{C}$ and $\mathrm{K} 8 \mathrm{C}$, respectively.

As a first approximation, one may assume that the distancedependent decay of the electric field strength from the SAM surface to the solution follows an exponential function according to

$$
|\vec{F}(x)|=-E_{\mathrm{SAM}} \kappa \exp (-\kappa x)
$$

where $E_{\mathrm{SAM}}$ is the potential at the SAM surface and $\kappa$ is the reciprocal Debye length.

The MD simulations allow determining the distance from the centre of the $\mathrm{C} \equiv \mathrm{N}$ bond to the SAM surface as well as the angle of the $\mathrm{C} \equiv \mathrm{N}$ bond with respect to the surface normal, i.e. the vector of the interfacial electric field. Averaging the respective parameters over the last $5 \mathrm{~ns}$ of a $10 \mathrm{~ns}$ production run, these quantities are evaluated to be $4.4 \mathrm{~nm}$ and $127^{\circ}$ for $\mathrm{K} 39 \mathrm{C}$, and $2.2 \mathrm{~nm}$ and $105^{\circ}$ for $\mathrm{K} 8 \mathrm{C}$. Combining eqn (3) and (4), one may then evaluate the ratio of the frequency difference $\Delta \nu(\mathrm{K} 39 \mathrm{C}) / \Delta \nu(\mathrm{K} 8 \mathrm{C})$. The quantity $E_{\mathrm{SAM}}$ cancels out. The resultant value is 0.78 and thus larger than the experimental ratio of 0.33 , implying that the theoretical approach overestimates the distance-dependent decay of the local electric field. The discrepancy may be mainly due to the underlying assumptions of eqn (4) that neglect spatially fixed charges in the diffuse double layer which are introduced by the immobilisation of Cyt-c at the SAM/solution interface. The consequences are particularly severe for the label at $\mathrm{K} 8 \mathrm{C}$ since it is in the vicinity of several positively charged lysine residues. These residues may reorient towards the negatively charged SAM surface, thereby modulating the electric field. Such effects are likely to be less severe for the more remote label in $\mathrm{K} 39 \mathrm{C}$, both due to the longer distance from the SAM surface and the lower number of nearby charged residues.

In conclusion, we have presented an approach that allows mapping the local electric field experienced by proteins immobilized at charged interfaces. Based on the comparative spectroscopic analysis of the protein in solution and in the immobilized state, it is possible to deconvolute contributions arising from the internal electrostatic field of the protein $\left|\vec{F}_{\text {protein }}\right|$ from the external field $|\vec{F}(x)|$ originating from the potential drop across the SAM/solution interface. Differences in $\left|\vec{F}_{\text {protein }}\right|$, determined for two solvent-exposed label positions, are attributed to the local arrangement of charged and polar residues which may in turn also modulate the interfacial electric field experienced by the protein immobilized on a charged surface. As a consequence, an exponential distance dependence of $|\vec{F}(x)|$ at the SAM/solution interface is insufficient for describing the local field strength for labels exposed to the diffuse double layer. The value of $|\vec{F}(x)|$ for the label position of $\mathrm{K} 8 \mathrm{C}$ is determined to be $9.4 \times 10^{8} \mathrm{~V} \mathrm{~m}^{-1}$, according to eqn (3) $\left(\alpha=105^{\circ}\right)$. This result agrees in order of magnitude with previous estimates. ${ }^{17}$

The present study demonstrates a proof-of-principle and thus constitutes a promising starting point for a future comprehensive investigation of the electric field distribution at the SAM/ protein interface, which is required for a quantitative analysis of the electric field effects on the structure and electron transfer dynamics of Cyt-c and other proteins at biomimetic interfaces.

\section{Notes and references}

1 R. J. Clarke, Adv. Colloid Interface Sci., 2001, 89, 263.

2 S. Geibel, T. Friedrich, P. Ormos, P. G. Wood, G. Nagel and E. Bamberg, Biophys. J., 2001, 81, 2059.

3 F. Bezanilla, Nat. Rev. Mol. Cell Biol., 2008, 9, 323; and references therein.

4 (a) A. Pilotelle-Bunner, P. Beaunier, J. Tandori, P. Maroti, R. J. Clarke and P. Sebban, Biochim. Biophys. Acta, Bioenerg., 2009, 1787, 1039; (b) Z. D. Popovic, G. J. Kovacs, P. S. Vincett, G. Alegria and P. L. Dutton, Chem. Phys., 1986, 110, 227; (c) S. Franzen, R. F. Goldstein and S. G. Boxer, J. Phys. Chem., 1990, 94, 5135 .

5 M. C. Koag and D. M. Papazian, Channels, 2009, 3, 356.

6 C. Weidemüller and K. Hauser, Biochim. Biophys. Acta, Bioenerg., 2009, 1787, 721, and references therein.

7 K. K. Ly, M. Sezer, N. Wisitruangsakul, J. J. Feng, A. Kranich, D. Millo, I. M. Weidinger, I. Zebger, D. H. Murgida and P. Hildebrandt, FEBS J., 2011, 278, 1382; and references therein.

8 I. T. Suydam, C. D. Snow, V. S. Pande and S. G. Boxer, Science, 2006, 313, 200.

9 I. T. Suydam and S. G. Boxer, Biochemistry, 2003, 42, 12050.

10 A. T. Fafarman, L. J. Webb, J. I. Chuang and S. G. Boxer, J. Am. Chem. Soc., 2006, 128, 13356.

11 A. T. Fafarman, P. A. Sigala, D. Herschlag and S. G. Boxer, J. Am. Chem. Soc., 2010, 132, 12811.

12 D. J. Aschaffenburg and R. S. Moog, J. Phys. Chem. B, 2009, 113, 12736.

13 N. Wisitruangsakul, I. Zebger, K. H. Ly, D. H. Murgida, S. Egkasit and P. Hildebrandt, Phys. Chem. Chem. Phys., 2008, 10, 5276

14 D. H. Murgida and P. Hildebrandt, Phys. Chem. Chem. Phys., $2005,7,3773$.

15 O. Rüdiger, J. M. Abad, E. C. Hatchikian, V. M. Fernandez and A. L. De Lacey, J. Am. Chem. Soc., 2005, 127, 16008.

16 D. Alvarez-Paggi, D. F. Martín, P. M. De Biase, P. Hildebrandt, M. A. Martí and D. H. Murgida, J. Am. Chem. Soc., 2010, 132, 5769 .

17 D. H. Murgida and P. Hildebrandt, J. Phys. Chem. B, 2001, 105, 1578. 